\title{
Effect of remote clouds on surface UV irradiance
}

\author{
M. Degünther, R. Meerkötter \\ Deutsches Zentrum für Luft- und Raumfahrt (DLR), Institut für Physik der Atmosphäre, \\ Oberpfaffenhofen, 82234 Wessling, Germany \\ e-mail: markus.deguenther@dir.de
}

Received: 15 October 1999 / Revised: 23 February 2000 / Accepted: 2 March 2000

\begin{abstract}
Clouds affect local surface UV irradiance, even if the horizontal distance from the radiation observation site amounts to several kilometers. In order to investigate this effect, which we call remote clouds effect, a 3-dimensional radiative transfer model is applied. Assuming the atmosphere is subdivided into a quadratic based sector and its surrounding, we quantify the influence of changing cloud coverage within this surrounding from $0 \%$ to $100 \%$ on surface UV irradiance at the sector center. To work out this remote clouds influence as a function of sector base size, we made some calculations for different sizes between $10 \mathrm{~km} \times 10 \mathrm{~km}$ and $100 \mathrm{~km} \times 100 \mathrm{~km}$. It appears that in the case of small sectors (base size $\leq 20 \mathrm{~km} \times 20 \mathrm{~km}$ ) the remote clouds effect is highly variable: Depending on cloud structure, solar zenith angle and wavelength, the surface UV irradiance may be enhanced up to $15 \%$ as well as reduced by more than $50 \%$. In contrast, for larger sectors it is always the case that enhancements become smaller by $5 \%$ if sector base size exceeds $60 \mathrm{~km} \times 60 \mathrm{~km}$. However, these values are upper estimates of the remote cloud effects and they are found only for special cloud structures. Since these structures might occur but cannot be regarded as typical, different satellite observed cloud formations (horizontal resolution about $1 \mathrm{~km} \times 1 \mathrm{~km}$ ) have also been investigated. For these more common cloud distributions we find remote cloud effects to be distinctly smaller than the corresponding upper estimates, e.g., for a sector with base size of $25 \mathrm{~km} \times 25 \mathrm{~km}$ the surface UV irradiance error due to ignoring the actual remote clouds and replacing their influence with periodic horizontal boundary conditions is less than $3 \%$, whereas the upper estimate of remote clouds effect would suggest an error close to $10 \%$.
\end{abstract}

Key words: Atmospheric composition and structure (transmission and scattering of radiation) - Meteorology and atmospheric dynamics (radiative process)

Correspondence to: M. Degünther

\section{Introduction}

Clouds have considerable impact on the ultraviolet (UV) radiation reaching the ground. As is known from measurements, interactions between UV radiation and clouds may result in surface UV irradiances ranging from less than $5 \%$ and to more than $125 \%$ of the clear sky value (Estupinan et al., 1996; Mims and Frederick, 1994). Clouds are clearly 3 -dimensional (3d) formations. Therefore, correct analysis of their influence on surface UV irradiance requires $3 \mathrm{~d}$ radiative transfer modeling in combination with a suitable cloud description, which may be derived e.g., from satellite data or large eddy simulations. In practice it is not possible to model the influence of arbitrary large broken cloud fields, but it is possible to consider a certain fraction of the cloudy sky. Thus, the uncertainty caused by this inevitable limitation has to be quantified for meaningful analyses of surface UV irradiance. Such a quantification is useful not only for modeling purposes, but also for interpretation of ground-based UV measurements.

Although many papers have investigated the effects of $3 \mathrm{~d}$ cloud structures on atmospheric radiative transfer (e.g., O'Hirok and Gautier, 1998a, b; Hignett and Taylor, 1996), the question still remains as to which part of a cloud field is of significance for surface UV irradiance. In most studies the influence of specific cloud distributions on the radiation field is investigated for a spatially limited atmospheric sector. The radiative interaction between this sector and the surrounding atmosphere is described by horizontal boundary conditions. Because it is impossible to specify the true horizontal boundary conditions (this would require an a priori knowledge of the radiative transfer within the whole atmosphere), they are frequently defined as specular or periodic. However, this definition implies an atmosphere of some regular structure, which generally is an unrealistic assumption for cloudy conditions (Zhu et al., 1992; Lee et al., 1994). Consequently, since the influence of the real cloud field outside the 
atmospheric sector, termed remote clouds, cannot be taken into account, but has to be replaced by horizontal boundary conditions, assumptions are introduced which could be perfectly wrong.

In order to investigate the impact of this potential source of error on surface UV irradiance, in the current study the effect of remote clouds is systematically analyzed and quantified by means of a $3 \mathrm{~d}$ radiative transfer model (Spherical Harmonics Discrete Ordinate Method or SHDOM, Evans, 1998). After describing the modeling procedure in Sect. 2, we present in Sect. 3 the remote clouds effect (RCE) on surface UV irradiance for different cloud constellations. Since the RCE is the superposition of different mechanisms that enhance as well as reduce surface UV irradiance, in a first step investigations focus on remote cloud distributions which maximize the effects of these mechanisms. Because calculations are made for atmospheric sectors of different size, the RCE dependence on the horizontal distance between observer and remote clouds is obtained. In a second step cloud fields as observed with the AVHRR instrument onboard the NOAA satellites (horizontal resolution about $1 \mathrm{~km} \times 1 \mathrm{~km})$ are studied. For each scene the error in surface UV irradiance due to neglecting the influence of the actual remote clouds is determined. Finally, in Sect. 4, we discuss the possible extrapolation of the results presented to conditions not explicitly considered in our model calculations.

\section{Modeling surface UV irradiance}

\subsection{Radiative transfer model}

In the radiative transfer model SHDOM the optical properties of the atmosphere and the surface reflection are specified at the points of a spatial grid and of a grid covering the ground, respectively. Radiances are calculated at the points of an additional spatial grid, which is not necessarily identical with the property grid, by integrating the equation of radiative transfer along discrete ordinates. This is done by assuming linear variation of the extinction coefficient and the product of extinction coefficient and source function inside each grid cell. The UV radiation field is determined, taking into account absorption by ozone and aerosol particles, as well as scattering by air molecules, cloud and aerosol particles.

In order to check the accuracy of SHDOM, in a first step comparisons with a matrix operator model (horizontally homogeneous atmosphere) (Meerkötter et al., 1997) and a 3d Monte Carlo model (broken clouds) (B. Mayer, 1999, private communication) were performed. The differences found for the calculated surface irradiances were around 3\% for wavelength $300 \mathrm{~nm}$ and around $1 \%$ for wavelength $330 \mathrm{~nm}$. In a second step the results of SHDOM were compared with measurements taken under clear sky conditions without snow coverage. The model results deviate by less than $6 \%(10 \%)$ from the measurements for wavelengths greater (smaller) than
$330 \mathrm{~nm}$ (Degünther et al., 1998) and the spectral dependence of the observed deviation is very similar to that found for 1d models (e.g., Mayer et al., 1997). Although the comparison between measured and calculated surface UV irradiances so far is encouraging, the general evaluation of SHDOM is still an outstanding issue.

\subsection{Model input}

The optical properties of the cloud-free atmosphere are derived from vertical profiles of ozone density and meteorological data as prescribed by a midlatitude standard atmosphere (McClatchey et al., 1972). The ozone profile is scaled to give a total of 300 DU. The aerosol optical depth is $0.2,0.33$ and 0.35 at $550 \mathrm{~nm}$, $330 \mathrm{~nm}$ and $300 \mathrm{~nm}$ wavelength, respectively, and the underlying aerosol profile corresponds to the continental model (CON-I) after WMO (1986). Cloud microphysics is described by lognormal droplet size distributions related to either stratocumulus or cumulus congestus type (Table 1), and it is assumed to be constant within the cloud. Depending on the respective problem, the atmospheric optical properties are specified in 21 to 24 different vertical levels with a horizontal resolution of $200 \mathrm{~m}$ to $250 \mathrm{~m}$. Finally, surface optical properties are described by a Lambertian reflector with albedo 0.03 . In the UV spectral range this value is a reasonable mean for snow-free surfaces excluding deserts and salt flats (Eck et al., 1987; Blumthaler and Ambach, 1988).

\section{Results}

The RCE on surface UV irradiance is essentially a superposition of the three mechanisms illustrated in Fig. 1, all of them depending in a different manner on cloud structure, wavelength and solar zenith angle $(S Z A)$. The first mechanism, the albedo effect, leads to an enhancement of surface UV irradiance if the surface is not snow covered. Photons which would have hit the surface under clear sky conditions are reflected by clouds and afterwards backscattered by the atmosphere above the clouds. Thus, instead of being absorbed at the surface, these photons contribute to a diffuse skylight enhancement. The second mechanism, termed scattering

Table 1. Microphysical parameters of cloud models. The droplet size distributions for the two models stratocumulus and cumulus congestus as proposed by McCartney (1976) are approximated using lognormal distributions. The respective relative distribution is obtained from mode radius $r_{m}$ and standard deviation $\sigma$, while droplet number concentration $N$ is scaled to the prescribed cloud optical depth

\begin{tabular}{llll}
\hline Cloud model & $r_{m}[\mu \mathrm{m}]$ & $\sigma[\mu \mathrm{m}]$ & $N$ \\
\hline Stratocumulus & 3.5 & 1.70 & $\begin{array}{r}\text { Scaled to given } \\
\text { optical depth }\end{array}$ \\
Cumulus congestus & 6.0 & 1.95 & \\
\hline
\end{tabular}



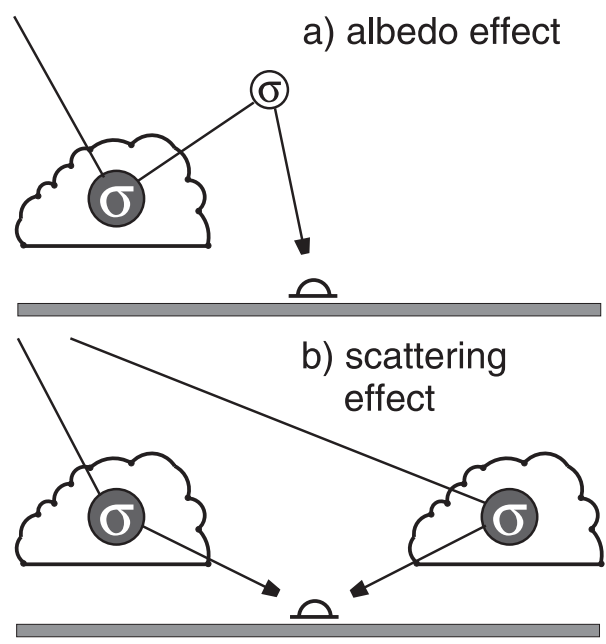

c) extinction effect

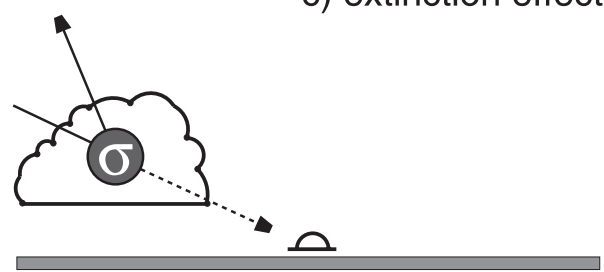

Fig. 1a-c. Influence of remote clouds on surface UV irradiance. The albedo and scattering effect enhance surface UV irradiance, whereas the extinction effect leads to a decrease. In cases $\mathbf{a}$ and $\mathbf{b}$ scattering of photons by remote clouds increase the diffuse skylight at the observation site, while in case $\mathbf{c}$ remote clouds redirect photons that otherwise would have reached the observer. The white $\sigma$ in the dark circle symbolizes cloud scattering, while the black $\sigma$ indicates atmospheric backscattering

effect, enhances the observed diffuse skylight as well, since scattering by clouds partly changes photon propagation directions towards the observer. However, cloud scattering may also result in deflection of photons, which would have reached the radiation observation site at the ground in the cloud-free case. This third mechanism attenuates surface UV irradiance and it is called the extinction effect.

In the following section two special configurations of remote clouds are investigated, allowing us to assess the impact of maximum attenuation and intensification effects on surface UV irradiance. Using two wavelengths and two $S Z A$ we show the relative significance of these effects varies with the distance between observer and remote clouds. In the second section we present the RCE as calculated for three different satellite observed, i.e., real, cloud scenes typical for scattered and broken clouds as well as for complete cloud coverage.

\subsection{Maximum surface UV irradiance attenuation and intensification by remote clouds}

To obtain maximum effects of the three described surface UV irradiance affecting mechanisms in dependence on remote clouds distance from the observer, firstly the atmosphere is assumed to be subdivided into a sector with base size $S \mathrm{~km} \times S \mathrm{~km}$ and its surrounding (Fig. 2). Secondly, sectors with different base sizes containing either the given cloud structures or being cloud-free are defined. Thirdly, for each constellation two $3 \mathrm{~d}$ radiative transfer simulations are carried out. In the first one there are no clouds outside the sector and as a result the effect of each single mechanism illustrated in Fig. 1 is zero. In the second simulation the atmospheric sector is assumed to be surrounded by a complete cloud cover. This ensures maximum attenuation and intensification of surface UV irradiance by the extinction effect and by the albedo plus scattering effect, respectively. Finally, ratios relating surface UV irradiances calculated for these two extreme conditions are determined. The ratio obtained for sector center $X$ (Fig. 2) is taken as a measure of the RCE which results from superposing maximum effects of the attenuation and intensification mechanisms.

In Fig. 3a-f this ratio is displayed as function of $S$ and sector base size for the two $S Z A 30^{\circ}$ and $60^{\circ}$ and the two wavelengths $300 \mathrm{~nm}$ and $330 \mathrm{~nm}$. The product $0.56 \cdot S$ can be interpreted as the mean distance between an observer located at sector center $X$ and the nearest remote clouds beyond the horizontal sector boundaries (Fig. 2). The two selected wavelengths represent radiation strongly absorbed by ozone as well as radiation not much affected by ozone absorption. Calculations are performed for cloud-free and homogeneously cloudfilled sectors. Clouds in the sector as well as the remote clouds are located within the same layer CL (Fig. 2). Figure. $3 \mathrm{a}-\mathrm{f}$ shows results for $\mathrm{CL}=1-2 \mathrm{~km}, \mathrm{CL}=$ 4-5 $\mathrm{km}$ and $\mathrm{CL}=1-5 \mathrm{~km}$, respectively. The microphysics of clouds with $1 \mathrm{~km}$ vertical extension is of stratocumulus type, whereas thick clouds between $1 \mathrm{~km}$ and $5 \mathrm{~km}$ height are assumed to be of cumulus congestus type (Table 1). All clouds have a uniform volume extinction coefficient of $20 \mathrm{~km}^{-1}$ (at $550 \mathrm{~nm}$ ).

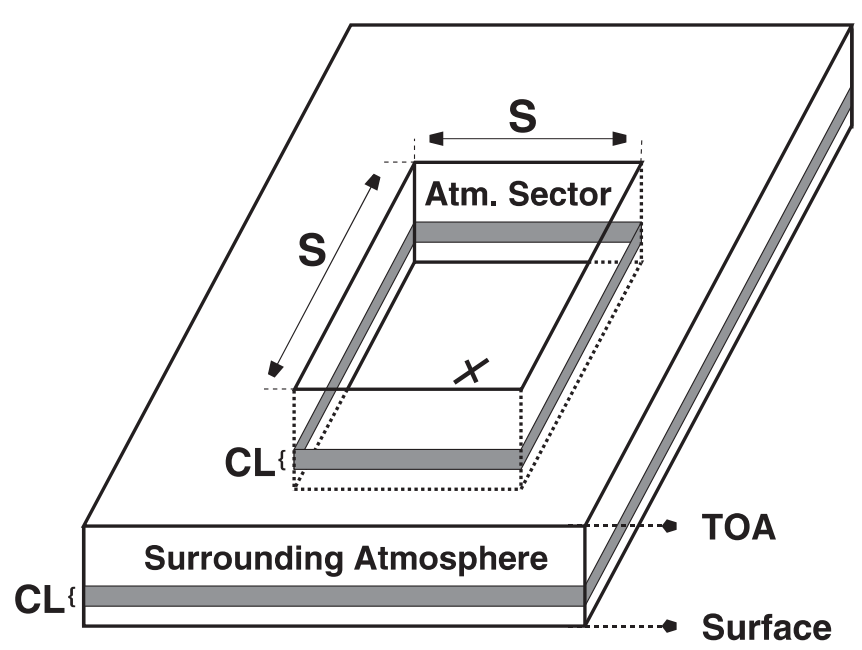

Fig. 2. Illustration of the atmospheric subdivision in a sector and its surrounding. The quadratic sector has a base size of $S \mathrm{~km} \times S \mathrm{~km}$ and is embedded in a horizontally infinite atmosphere. Base and top of clouds in the sector as well as in the surrounding atmosphere are identical. The vertical cloud extension is denoted by $C L$. $X$ marks sector center. TOA is the abbreviation for top-of-atmosphere 


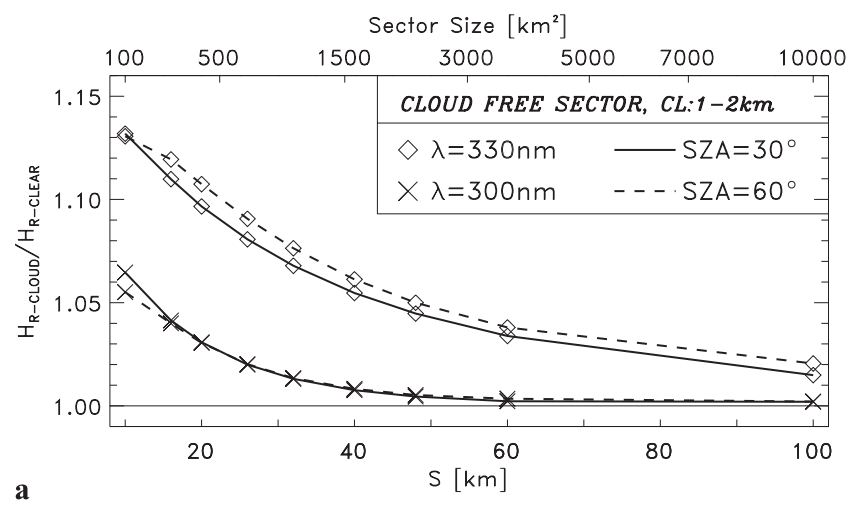

a
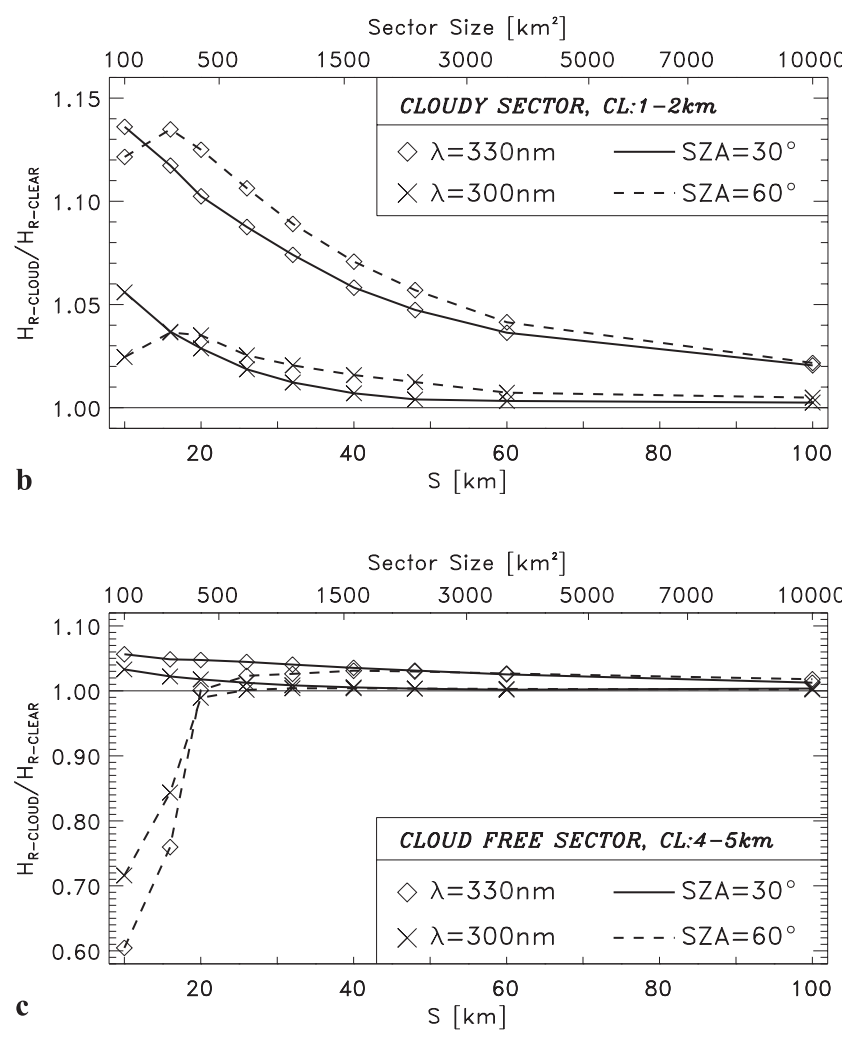

Fig. 3a-f. Ratio of surface irradiances at sector center calculated with $\left(H_{R-C l o u d}\right)$ and without $\left(H_{R-C l e a r}\right)$ remote clouds as a function of $S$ and of sector base size. This ratio is used to quantify the RCE. Calculations are performed for homogeneously cloud-filled as well as for cloud-free sectors taking into account two $S Z A\left(30^{\circ}, 60^{\circ}\right)$ and two wavelengths $(300 \mathrm{~nm}, 330 \mathrm{~nm})$. Clouds in and outside the sector are

CL = 1-2 km (Fig. 3a, b). Remote clouds enhance surface UV irradiance for all sector sizes in the range from $S=10$ to $S=100 \mathrm{~km}$. Thus, the scattering and in particular the albedo effect outweigh the extinction effect. The RCE depends more strongly on wavelength than on $S Z A$. This is for two reasons: firstly, the atmospheric absorption above the clouds is stronger for the wavelength $300 \mathrm{~nm}$. Therefore, considerably less radiation reflected by the clouds is backscattered. Secondly, the spectral atmospheric transmittance is smaller for $300 \mathrm{~nm}$ not only in the stratospheric ozone layer, but also in the troposphere. Consequently, less of the radiation scattered by remote clouds reaches the sector center later and, in addition, the radiation extinction by remote
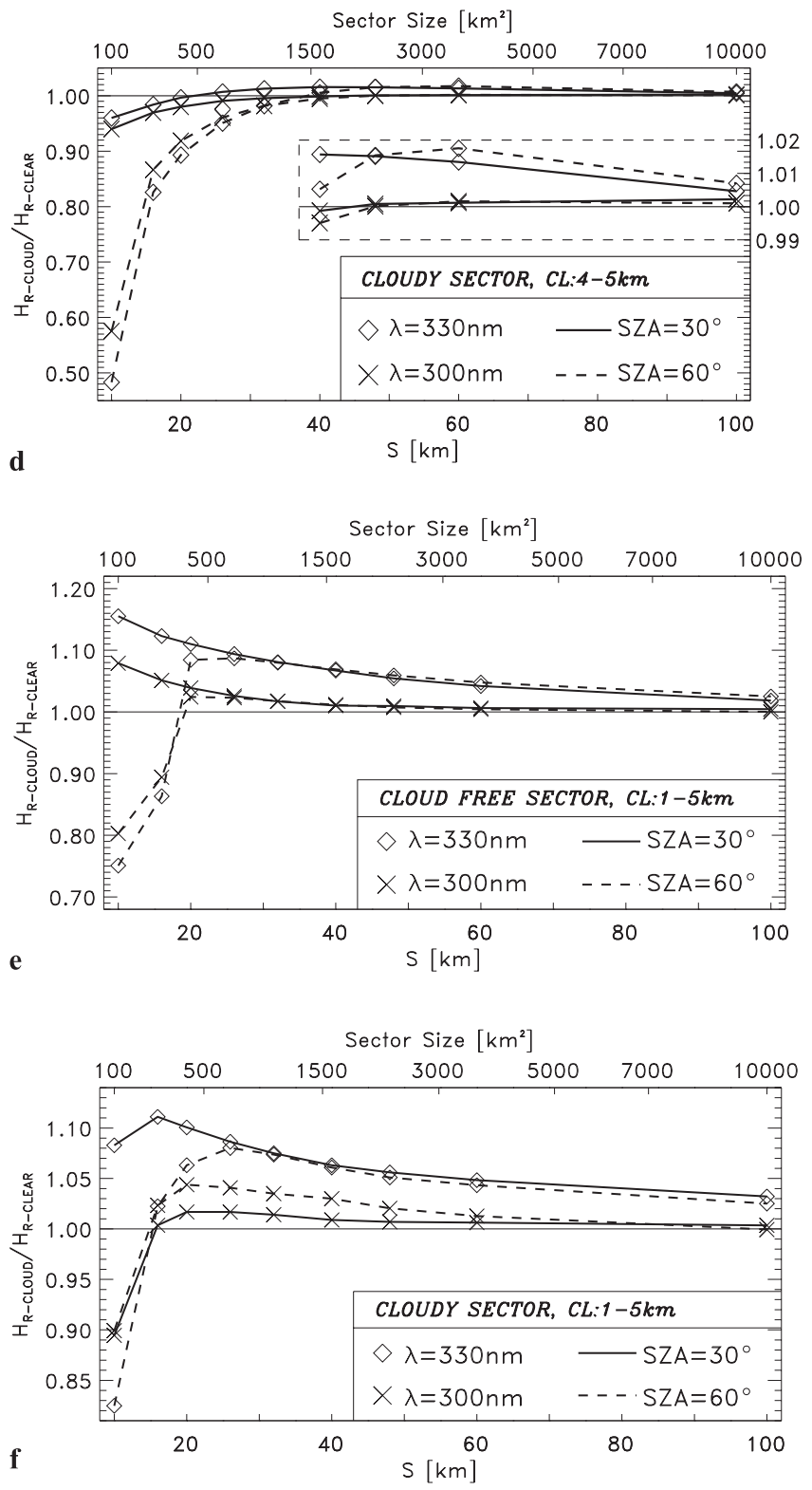

embedded within layer CL (Fig. 2). Results are presented for $\mathbf{a}, \mathbf{b}$ $\mathrm{CL}=1-2 \mathrm{~km}, \mathbf{c}, \mathbf{d} \mathrm{CL}=4-5 \mathrm{~km}$ and $\mathbf{e}, \mathbf{f} \mathrm{CL}=1-5 \mathrm{~km}$. The mean distance between the observer at sector center and the nearest remote clouds is $0.56 \cdot S$. The inset in Fig. $3 \mathrm{~d}$ shows the ratio $H_{R-\text { Cloud }} / H_{R-\text { Clear }}$ for $S \geq 40 \mathrm{~km}$ on a zoomed scale

clouds is of minor importance. Although the RCE dependence on $S$ is very similar for $S Z A=30^{\circ}$ and $S Z A=60^{\circ}$, differences are found for small sector sizes $(\mathrm{S} \leq 16 \mathrm{~km})$. The reason is that with a low Sun angle more radiation incidents with larger zenith angles and occur e.g., for $S=10 \mathrm{~km}$ the surrounding remote clouds shade radiation with zenith angles larger than about $70^{\circ}$. Therefore, extinction effectively counteracts the scattering and albedo effect. Furthermore, radiation laterally penetrating into the sector below layer $\mathrm{CL}$ is more efficiently 'trapped' under clouds, and hence has a stronger impact on surface UV irradiance. Thus, extinction of this radiation by remote clouds is especially effective if the Sun is low and the sector small and, moreover; 
CL $=$ 4-5 km (Fig. 3c, d). In the case of small sectors and low Sun remote clouds decrease surface UV irradiance. For $S=10 \mathrm{~km}(S=16 \mathrm{~km})$ about $65 \%$ $(50 \%)$ of the sky as observed from the sector center is covered with remote clouds. Thus, under low Sun conditions not only the direct, but also a significant part of the diffuse radiation is shaded leading to a clearly dominating extinction effect. Furthermore, due to the 'trapping effect' described, extinction is dominant for small clouded sectors even in combination with a high Sun angle $\left(S Z A=30^{\circ}\right)$. The predominance of the extinction effect is further supported by a comparatively small albedo effect. Because the atmospheric layer above the clouds has a smaller optical depth than e.g., in the case $\mathrm{CL}=1-2 \mathrm{~km}$, less cloud reflected radiation is backscattered. This albedo effect reduction becomes particularly obvious where the extinction effect is negligible, i.e., for large sectors. In that case surface UV irradiance enhancements are distinctly smaller than for clouds embedded between $1 \mathrm{~km}$ and $2 \mathrm{~km}$. Since the extinction effect is especially marked in the range of small $S$, the RCE depends more strongly on SZA than on wavelength. However, for larger $S$ where the albedo effect becomes dominant, the RCE varies with wavelength but is nearly independent of $S Z A$. Finally, the extinction as well as the albedo effect is more pronounced for wavelength $330 \mathrm{~nm}$ when compared to $300 \mathrm{~nm}$. Therefore, in the case of low Sun with prevailing extinction for small $S$ and dominating albedo effect for larger $S$, the two dashed curves representing the RCE dependence on $S$ for $S Z A=60^{\circ}$ and wavelengths $300 \mathrm{~nm}$ and $330 \mathrm{~nm}$ must have a cross-over point close to $H_{R-\text { Cloud }} / H_{R-\text { Clear }}=1$.

CL $=$ 1-5 km (Fig. 3e, f). With a vertical extension of $4 \mathrm{~km}$ the clouds have four times the geometrical depth of the clouds in the cases $\mathrm{CL}=1-2 \mathrm{~km}$ and $\mathrm{CL}=4$ $5 \mathrm{~km}$. Hence, especially for small sectors, illumination of the clouds vertical interfaces plays an important role. Regarding cloud-free sectors, RCE depends in a very similar manner on $S$ as found for CL $=4-5 \mathrm{~km}$ for both wavelengths and $S Z A$. Because of the greater geometrical depth of remote clouds backscattering at their vertical interfaces is enhanced and in particular for small sectors the ratio $H_{R-C l o u d} / H_{R-\text { Clear }}$ is to some degree higher. Furthermore, a greater cloud optical depth increases their reflectivity resulting in a stronger albedo effect. For small clouded sectors $(S=10 \mathrm{~km})$ the horizontal distance between sector center $X$ and its edge is comparable to cloud geometrical depth. Therefore, the contribution of radiation entering the sector cloud through its vertical interfaces is important and the extinction of this radiation by remote clouds leads to a distinct decrease of surface UV irradiance. For wavelength $300 \mathrm{~nm}$ as well as for $330 \mathrm{~nm}$ in combination with low Sun this radiation attenuation is the dominating effect. By contrast, when $S Z A=30^{\circ}$ and wavelength $330 \mathrm{~nm}$ the ratio $H_{R-\text { Cloud }} / H_{R-\text { Clear }}$ is greater than 1 , which means that the sum of scattering and albedo effect still outweighs extinction. Increasing $S$ to more than about $20-25 \mathrm{~km}$ results in a predominance of the albedo effect causing a ratio $H_{R-\text { Cloud }} / H_{R-\text { Clear }}$ greater than 1 for all $S Z A$-wavelength combinations and a stronger dependence of RCE.

Table 2 gives an idea which section of a cloudy sky has to be taken into account in order to avoid RCE exceeding a given limit. It illustrates, that for higher clouds $(\mathrm{CL}=4-5 \mathrm{~km})$ the RCE decreases quickly with increasing $S$. A sector size of $30 \mathrm{~km} \times 30 \mathrm{~km}$ suffices to keep maximum influence by remote clouds on surface UV irradiance clearly below 5\%. With lower stratus $(\mathrm{CL}=1-2 \mathrm{~km}) \mathrm{RCE}$ is considerably lower for small sectors, but it decreases only slowly with sector enlargement. Consequently, in order to keep the maximum RCE smaller than $5 \%$, the cloud distribution in a sector of $2500 \mathrm{~km}^{2}$ has to be taken into account. Finally, for $\mathrm{CL}=1-5 \mathrm{~km}$ the maximum effects for small sectors are similar to those found for $\mathrm{CL}=4-5 \mathrm{~km}$, whereas for larger $S$ they become comparable to the effects found for stratus clouds between $1 \mathrm{~km}$ and $2 \mathrm{~km}$ above ground. This is explained by the fact, that for small sectors the extinction of diffuse and eventually direct radiation is very pronounced with high cloud tops and therefore dominates RCE. For larger sectors the magnitude of the albedo effect is decisive. On the one hand, when compared to $\mathrm{CL}=1-2 \mathrm{~km}$, the larger cloud optical depth for $\mathrm{CL}=1-5 \mathrm{~km}$ enhances cloud reflectivity, on the other hand the smaller optical depth of the atmosphere above the clouds leads to less backscattering of cloud reflected radiation. Thus, for $C L=1-2 \mathrm{~km}$ and for $\mathrm{CL}=1-5 \mathrm{~km}$ the albedo effect is similar resulting in similar RCE for large sectors.

\subsection{Remote clouds effect in satellite observed cloud scenes}

As already mentioned it is impossible to investigate the influence of arbitrary large $3 \mathrm{~d}$ structured cloud fields on atmospheric radiation. Only that part within a spatially limited atmospheric sector can be taken into account and RCE has to be replaced by highly uncertain horizontal boundary conditions. In order to assess how this uncertainty affects surface UV irradiance for typical cloud structures, we have looked at sectors of $25 \mathrm{~km} \times 25 \mathrm{~km}$. This base size is large enough to ensure RCE below $10 \%$ (Table 2) and simultaneously it is small enough to keep the required CPU-time for $3 \mathrm{~d}$

Table 2. Minimum sector sizes $S$ required to avoid RCE exceeding a given maximum. If heights of cloud base and top are unknown the maximum of the $S$ values obtained for the three different cloud layers $(\mathrm{CL}=1-2 \mathrm{~km}, \mathrm{CL}=4-5 \mathrm{~km}, \mathrm{CL}=1-5 \mathrm{~km})$ is assumed

\begin{tabular}{rlrrl}
\hline RCE $(\%)$ & \multicolumn{4}{l}{ CL $[\mathrm{km}]$} \\
\cline { 2 - 4 } & $1-2$ & $4-5$ & $1-5$ & Unknown \\
\hline & \multicolumn{4}{l}{ Minimum sector size $S[\mathrm{~km}]$} \\
20 & $<10$ & 17 & 12 & 17 \\
15 & $<10$ & 19 & 15 & 19 \\
10 & 24 & 22 & 25 & 25 \\
5 & 50 & 28 & 60 & 60 \\
\hline
\end{tabular}




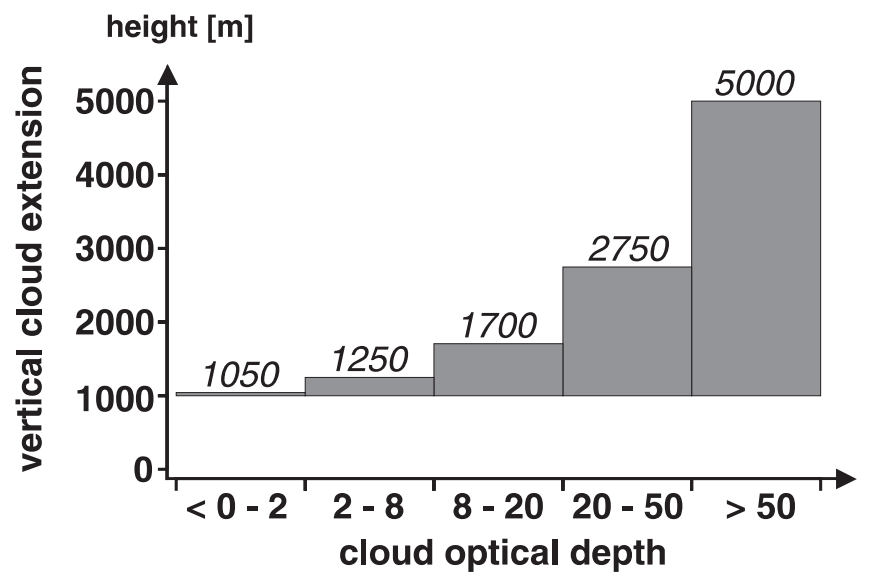

Fig. 4. Correlation between optical and geometrical cloud depth. In the case of satellite observations the cloud base is assumed to be uniformly $1 \mathrm{~km}$ above ground, whereas the top heights (given by the italic numbers) depends on the optical depth class to which the cloud belongs. The vertical cloud extensions are chosen to ensure a cloud extinction coefficient of $20 \mathrm{~km}^{-1}$ (at $550 \mathrm{~nm}$ ) for the mean of each optical depth class

radiative transfer calculations with reasonable spatial resolution in the order of hours. The distribution of cloud optical depth within the sector is derived from NOAA/AVHRR satellite data using the APOLLO algorithm (Kriebel et al., 1989). The cloud base is assumed to be $1 \mathrm{~km}$ above the ground, whereas cloud top height depends on cloud optical depth as illustrated in Fig. 4. The investigated cloud scenes were observed on July 01, 1996, at about 13 UTC over southern Germany. The corresponding $S Z A$ and solar azimuth angle is $31.3^{\circ}$ and $211.0^{\circ}$ (southwest), respectively. As in the foregoing section two $3 \mathrm{~d}$ radiative transfer model simulations are performed for each sector. In the first simulation the horizontal boundary conditions are periodic, i.e., a photon leaving the sector through one of its vertical bounding planes is assumed to re-enter through the opposite plane. Thus, the actual RCE is neglected and the radiative transfer is calculated as if the whole atmosphere consisted of equal sized sectors each containing identical the same cloud distribution as its neighbours. By contrast, in the second simulation the real (satellite observed) cloud field that surrounds the investigated atmospheric sector is considered. From both simulations we obtain an average of surface UV irradiance over an area of $4 \mathrm{~km} \times 4 \mathrm{~km}$ located around the sector center. To quantify RCE the ratio calculated from these two averages is considered.

Figure $5 \mathrm{a}-\mathrm{c}$ shows three distributions of cloud optical depth. The sectors represent situations with scattered clouds (Fig. 5a), broken clouds (Fig. 5b) and complete cloud coverage (Fig. 5c). For Fig. 5a-c the $\mathrm{RCE}$ is $1.2 \%, 2.5 \%$ and $2.2 \%$, respectively. These values are calculated for the wavelength $330 \mathrm{~nm}$, but the numbers are very similar for the wavelength $300 \mathrm{~nm}$. The smaller effect for Fig. 5a can be explained by the fact that direct radiation, which of course is not affected by remote clouds, significantly contributes to surface UV irradiance. Looking only at the diffuse component,
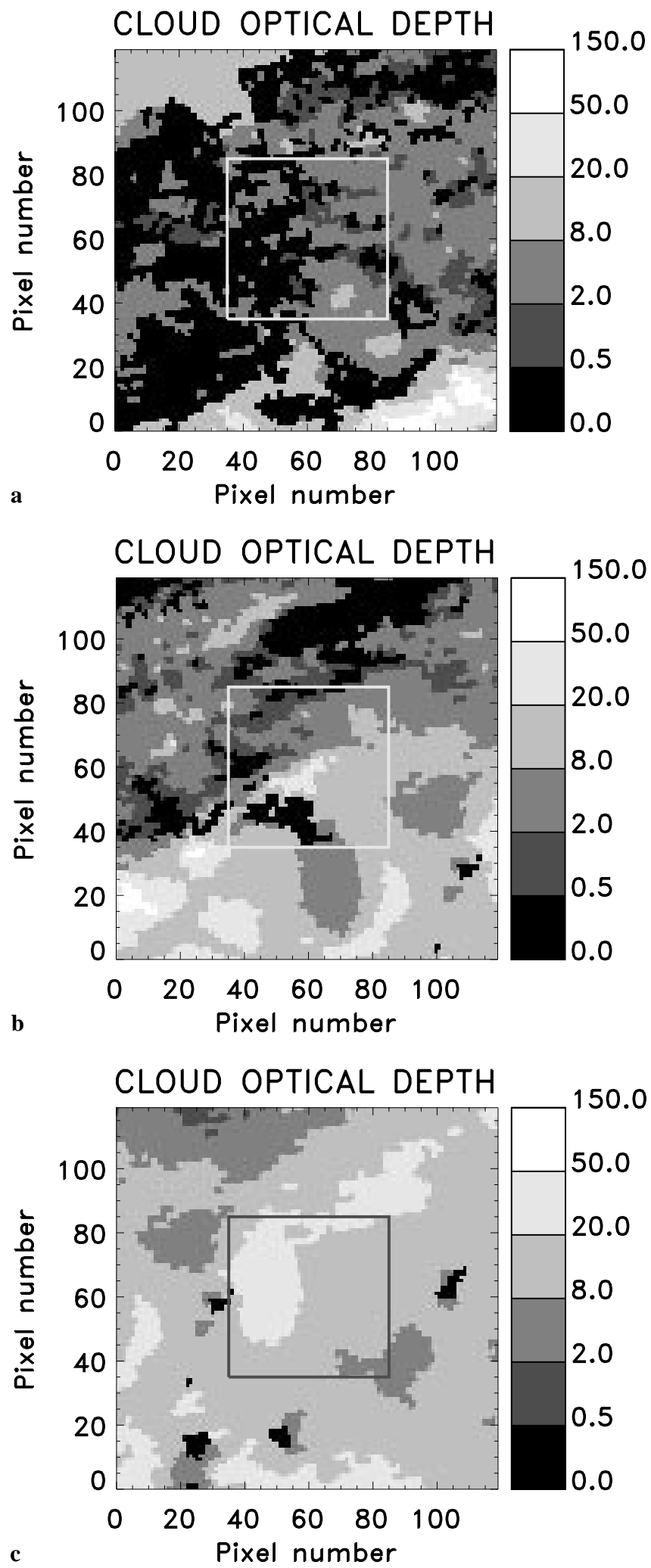

Fig. 5a-c. Cloud optical depth distributions obtained from NOAA/ AVHRR data using the APOLLO algorithm (Kriebel et al., 1989). Due to remapping the pixel size is $500 \mathrm{~m} \times 500 \mathrm{~m}$. All three scenes a, b and c are observed on July 01, 1997, over southern Germany at about 13 UTC. The Sun is located in the southwest, i.e., the lower left image corner. The square within each scene marks the atmospheric sector having a horizontal extension of $25 \mathrm{~km} \times 25 \mathrm{~km}$, whereas the clouds outside are the remote clouds 
remote clouds induced effects are comparable to those in Fig. $5 b$ and c. From these comparisons it can be inferred, that replacing the actual remote clouds by periodic horizontal boundary conditions results in surface UV irradiance errors no larger than about $2.5 \%$. This is valid for scattered and broken clouds as well as for complete cloud coverage. Thus, for the selected satellite observed scenes the RCE is considerably smaller than the corresponding value which is obtained assuming the effects of attenuation and intensification mechanisms to be maximum. Nevertheless, although for sectors with base size of $25 \mathrm{~km} \times 25 \mathrm{~km}$ cloud distributions as discussed in the preceding section are seldom seen, they might occur. Consequently, the occurrence of RCE distinctly larger than $2.5 \%$ cannot be excluded.

\section{Summary and concluding remarks}

The influence of remote clouds on surface UV irradiance has been investigated using a $3 \mathrm{~d}$ radiative transfer model. Firstly, special remote cloud configurations are taken into account, ensuring maximum effects of the surface UV irradiance attenuation and intensification mechanisms. The RCE is obtained as a function of atmospheric sector base size for the two different $S Z A$ $30^{\circ}$ and $60^{\circ}$, for the two different wavelengths $300 \mathrm{~nm}$ and $330 \mathrm{~nm}$ and for different cloud structures within the sector. Secondly the RCE is determined for real cloud scenes as observed with the AVHRR instrument onboard the NOAA satellites.

For small sectors $(S \leq 15-20 \mathrm{~km})$ the RCE on surface UV irradiance is very important and may be highly variable. Depending on wavelength, $S Z A$ and vertical cloud extension, surface UV irradiance may be enhanced up to $15 \%$ as well as reduced by more than $50 \%$. Therefore, for small sectors the maximum errors induced by neglecting RCE and the errors due to uncertainties of parameters describing the atmospheric optical properties (Schwander et al., 1997) are comparable. In larger sectors remote clouds have less influence but, in all cases, they do enhance surface UV irradiance. The influence is smallest for remote clouds with low or moderate optical depths and tops at high altitudes. In all cases the RCE is below 5\% for sectors with base of $60 \mathrm{~km} \times 60 \mathrm{~km}$ or larger.

Since cloud formations leading to maximum effects of the three described surface UV irradiance affecting mechanisms are not common, we also investigated satellite observed cloud scenes. These scenes represent broken and scattered clouds as well as complete cloud coverage within a sector of $25 \mathrm{~km} \times 25 \mathrm{~km}$. The $3 \mathrm{~d}$ model simulations show that replacing the actual remote clouds by periodic horizontal boundary conditions causes surface UV irradiance errors not greater than about $2-3 \%$. Thus, for atmospheric sectors with base size $25 \mathrm{~km} \times 25 \mathrm{~km}$ the RCE is in general distinctly less than the corresponding potential maximum, and consequently this sector size normally should suffice to reliably analyze ground results with satisfactory accuracy. Nevertheless, although an atmospheric sector of
$25 \mathrm{~km} \times 25 \mathrm{~km}$ does not guarantee negligible RCE, in each case the structure of the respective cloud field has to be carefully analyzed.

The results presented are valid for a plane surface with low albedo, (i.e., no snow coverage), for an ozone column of 300 DU and for one specific aerosol optical depth. Provided the UV radiation observation side is not shaded due to orography and no great altitude differences exist, the assumption of a plane surface is a good approximation. Since ground reflected photons do not contribute significantly to the observed UV irradiance in case of low surface albedo, eventually surface slopes and peaks are of minor importance. In contrast, a snowcovered surface (albedo $\geq 0.6-0.8$ ) leads to very different results, since the albedo effect is completely different and multiple reflection between surface and atmosphere/ clouds may become important. Finally, enhancing the tropospheric ozone and aerosol content reduces the RCE. This is because the tropospheric extinction is increased and less of the radiation scattered by remote clouds reaches the observer afterwards.

Despite the unavoidable restriction to a limited number of $S Z A$, wavelengths and atmospheric/surface conditions investigated, the results presented can be regarded as representative for a broad variety of snowfree conditions. They may be helpful to other experimentators in making meaningful data analysis and interpretation. Furthermore, the errors of $3 \mathrm{~d}$ radiative transfer calculations induced by inadequate description of RCE can be estimated.

Acknowledgements. This study was supported by the German Federal Ministry of Education, Science, Research and Technology (BMBF) within the ozone research program. A reviewer is thanked for comments and suggestions which helped to improve the clarity of the work.

Topical Editor J.-P. Duvel thanks C. Gautier and M.F. Bréon for their help in evaluating this paper.

\section{References}

Blumthaler, M., and W. Ambach, Solar UVB-albedo of various surfaces, Photochem. Photobiol., 48, 85-88, 1988.

Degünther, M., R. Meerkötter, A. Albold, and G. Seckmeyer, Case study on the influence of inhomogeneous surface albedo on UV irradiance, Geophys. Res. Letts, 25, 3587-3590, 1998.

Eck, T. F., P. K. Bhartia, P. H. Hwang, and L. L. Stowe, Reflectivity of earth's surface and clouds in ultraviolet from satellite observations, J. Geophys. Res., 92, 4287-4296, 1987.

Estupinan, J. G., S. Raman, G. H. Crescenti, J. J. Streicher, and W. F. Barnard, Effects of clouds and haze on UV-B radiation, J. Geophys. Res., 101, 16 807-16 816, 1996.

Evans, K. F., The spherical harmonics discrete ordinate method for three-dimensional atmospheric radiative transfer, J. Atmos. Sci., 55, 429-446, 1998.

Hignett, P., and J. P. Taylor, The radiative properties of inhomogeneous boundary layer cloud: Observations and modelling, QJR Meteorol. Soc., 122, 1341-1364, 1996.

Kriebel, K. T., R. W. Saunders, and G. Gesell, Optical properties of clouds derieved from fully cloudy AVHRR pixels, Beitr. Phys. Atmos., 62, 165-171, 1989.

Lee, J., J. Chou, R. C. Weger, and R. M. Welch, Clustering, randomness, and regularity in cloud fields. 4. Stratocumulus cloud fields, J. Geophys. Res., 99, 14 461-14 480, 1994. 
Mayer, B., G. Seckmeyer, and A. Kylling, Systematic long-term comparison of spectral UV measurements and UVSPEC modeling results, J. Geophys. Res., 102, 8755-8767, 1997.

McCartney, E. J., Optics of the atmosphere, Wiley and Sons, New York, 1976.

McClatchey, R. A., R. W. Fenn, J. E. A. Selby, F. E. Volz, and J. S. Garing, Optical properties of the atmosphere, Air Force System Command, US Air Force, AFCRL-72-0497, 1972.

Meerkötter, R., B. Wissinger, and G. Seckmeyer, Surface UV from ERS-2/GOME and NOAA/AVHRR data: a case study, Geophys. Res. Letts, 24, 1939-1942, 1997.

Mims, F. M., and J. E. Frederick, Cumulus clouds and UV-B, Nature, 371, 291, 1994

O'Hirok, W., and C. Gautier, A three-dimensional radiative transfer model to investigate the solar radiation within a cloudy atmosphere. Part I: spatial effects, J. Atmos. Sci., 55, 2162-2179, 1998a.

O'Hirok, W., and C. Gautier, A three-dimensional radiative transfer model to investigate the solar radiation within a cloudy atmosphere. Part II: Spectral effects, J. Atmos. Sci., 55, 30653076, 1998b.

Schwander, H., P. Koepke, and A. Ruggaber, Uncertainties in modeled UV irradiances due to limited accuracy and availability of input data, J. Geophys. Res., 102, 9419-9429, 1997.

WMO, A preliminary cloudless standard atmosphere for radiation computation, World Climate Research Programs, WMO/TD24, 1986.

Zhu, T., J. Lee, R. C. Weger, and R. M. Welch, Clustering, randomness, and regularity in cloud fields. 2. Cumulus cloud fields, J. Geophys. Res., 97, 20 537-20 558, 1992. 